\title{
Modified exorotation graft tension for tibial fixation in anterior cruciate ligament reconstruction: a randomized controlled trial
}

\author{
Ludwig A.P. Pontoh, ${ }^{1}$ Ismail H. Dilogo, ${ }^{2}$ Saptawati Bardosono, ${ }^{3}$ Andri M.T. Lubis, ${ }^{2}$ Alida R. Harahap, ${ }^{4}$ Jacub \\ Pandelaki, ${ }^{5}$ Mohammad Hidayat ${ }^{6}$ \\ ${ }^{1}$ Department of Orthopaedic and Traumatology, Faculty of Medicine, Universitas Indonesia, Fatmawati Hospital, Jakarta, Indonesia \\ ${ }^{2}$ Department of Orthopaedic and Traumatology, Faculty of Medicine, Universitas Indonesia, Cipto Mangunkusumo Hospital, \\ Jakarta, Indonesia \\ ${ }^{3}$ Department of Nutrition, Faculty of Medicine, Universitas Indonesia, Cipto Mangunkusumo Hospital, Jakarta, Indonesia \\ ${ }^{4}$ Eijkman Biomolecular Institute, Jakarta, Indonesia \\ ${ }^{5}$ Department of Radiology, Faculty of Medicine, Universitas Indonesia, Cipto Mangunkusumo Hospital, Jakarta, Indonesia \\ ${ }^{6}$ Department of Orthopaedic and Traumatology, Faculty of Medicine, Universitas Brawijaya, Saiful Anwar Hospital, Malang, \\ Indonesia
}

\section{ABSTRACT}

Background: The inability of anterior cruciate ligament reconstruction (ACLR) surgery to reduce tibial internal rotation causes many problems. A large tibial internal rotation will result in a patellofemoral pain syndrome. This study aimed to introduce a new technique of modified exorotation graft tension at tibial fixation to minimize endorotation, reduce tibial internal rotation, and prevent patellofemoral pain syndrome.

Methods: This study was a randomized double-blind controlled clinical trial. ACL rupture patients underwent ACLR surgery between December 2014 and Februrary 2015. They were randomized to standard endorotation group or modified exorotation group. Hamstring autograft was used and fixed with an EndoButton ${ }^{\circledR}$ and bioabsorbable interference screw. Tibial tubercle to trochlear grove (TTTG) was used to evaluate rotation, whereas cartilage oligomeric matrix protein (COMP) was employed to analyze cartilage breakdown. The functional outcome was measured using the Kujala score to assess patellofemoral joint function. Evaluations were conducted before the surgery and at 6 months postoperation.

Results: A total of 29 subjects were recruited. Sixteen subjects were allocated into the standard endorotation group, and 13 were assigned to the modified exorotation group. The exorotation group demonstrated better results than the endorotation group in all three parameters: TTTG ( $p=0.028)$, COMP $(p<0.001)$, and Kujala score $(p=0.015)$.

Conclusion: A new technique of modified exorotation direction of graft tension for ACL reconstructive surgery at tibial fixation showed a significant reduction in tibial internal rotation and cartilage breakdown. The proposed method could significantly improve the functional outcome of those with total ACL rupture.

Keywords: ACL reconstruction surgery, anterior cruciate ligament, cartilage oligomeric matrix protein, Kujala score, tibial tubercle trochlear groove

pISSN: 0853-1773• eISSN: 2252-8083• https://doi.org/10.13181/mji.v27i3.1765・Med J Indones. 2018;27:169-77

- Received 10 Jan $2017 \cdot$ Accepted 11 Apr 2018

Corresponding author: Ismail H. Dilogo

ismailortho@gmail.com

Copyright @ 2018 Authors. This is an open access article distributed under the terms of the Creative Commons Attribution-NonCommercial 4.0 International License (http://creativecommons.org/licenses/by-nc/4.0/), which permits unrestricted non-commercial use, distribution, and reproduction in any medium, provided the original author and source are properly cited. 
Anterior cruciate ligament (ACL) is a knee intraarticular ligament that serves as a primary static stabilizer of the joint. ACL rupture is commonly found in sports injuries, with annual incidence of 35 per 100,000 people. $^{1}$ This condition has serious consequences for the injured athlete as well as the generation population, as there is an increased risk of premature osteoarthritis, despite being treated. ${ }^{2,3}$ Moreover, it has been reported that ACL is the most common knee ligament injury requiring surgical reconstruction. ${ }^{3,4}$

There are numerous reasons for ACL failure. ${ }^{5}$ One of the most prominent factors in the failure of treatment is patellofemoral joint dysfunction, which presents with pain around the knee cap that aggravates with activities and often results in crepitation, swelling, and joint stiffness. In our country, where Islam is the main religion, patellofemoral joint dysfunction is further emphasized because the associated pain often limits the knees' full range of motion required for praying.

Several efforts have been made to improve patellofemoraljointfunction:modification oftunnel placement to improve the knee biomechanics, reenactment of double bundle reconstruction to restore the ligament anatomically, and acceleration of the postoperative rehabilitation program to provide a tailored timeline for return to activity. However, methods that have been proven to improve a patient's functional outcome postoperatively are lacking. ${ }^{6}$

Alteration in knee joint biomechanics after ACL injury is one of the defining factors for unsatisfying postoperative outcome. In ACL-deficient knees, the tibia is endorotated in relation to the femur. In the standard ACLR procedure, the tendon graft is further tensioned in the direction of endorotation, so that the altered relationship of the tibia and femur is uncorrected. Excessive tibial endorotation can lead to malaligment of the patellofemoral joint and result in patellofemoral joint pain, which prevents a satisfactory functional outcome after ACLR. ${ }^{7}$ In the present study, ACLR was modified by moving the direction of graft tensioning at the tibial fixation from standard endorotation to exorotation. The modification of graft tensioning direction was expected to correct the functional outcomes of patellofemoral malalignment and result in improved patellofemoral functions. This study is the first to modify graft tensioning direction for improved functional outcome after ACLR.

\section{METHODS}

This study was a randomized doubleblind clinical trial on two parallel groups of patients with total ACL rupture who underwent ACLR surgery between December 2014 and February 2015. The inclusion criteria were male or female aged less than 40 years old, with total ACL rupture as indicated by the history of injury, Lachman test +3 and magnetic resonance imaging (MRI) showing total rupture; and injury time between 1 and 12 months. The exclusion criteria were flexion contracture more than $20^{\circ}$, presence of anterior knee pain before surgery, presence of crepitation on patella movement, $Q$ angle $>14^{\circ}$ in males and $>17^{\circ}$ in females, thigh circumference $>2$ $\mathrm{cm}$, patellar maltracking, muscular dysfunction, rupture of other ligaments, and presence of patellar shift or tilt.

Of the 67 subjects who underwent ACLR surgery during the time frame, 55 subjects fulfilled the inclusion and exclusion criteria. However, only 49 subjects gave their consent. These 49 subjects were randomly allocated into two groups: the first group $(\mathrm{n}=25)$ used a standard endorotation tension direction, and the second group $(n=24)$ involved the modified exorotation tension direction. Throughout the clinical trial, 20 subjects were dropped due to one of the following reasons: subjects did not return for the required follow up, failure in joint aspiration, subjects opted for alternative treatment, and presence of repeat injury. A total of 29 subjects were included in the study: 16 subjects in the standard endorotation group and 13 subjects in the modified exorotation group (Figure 1).

\section{Surgical technique}

All patients underwent ACLR via arthroscopy using ipsilateral hamstring tendon autograft. In the control group, the tendon graft was tensioned into endorotation at $45^{\circ}$ in relation to the tibial anterior crest line. The tendon graft was then fixated to the tibial side using BioScrew ${ }^{\circledR}$ on the lateral side (Figure 1). In the modified 
Subjects undergoing ACLR in

Pondok Indah Hospital Dec

2014 - Feb 2015 (n=67)

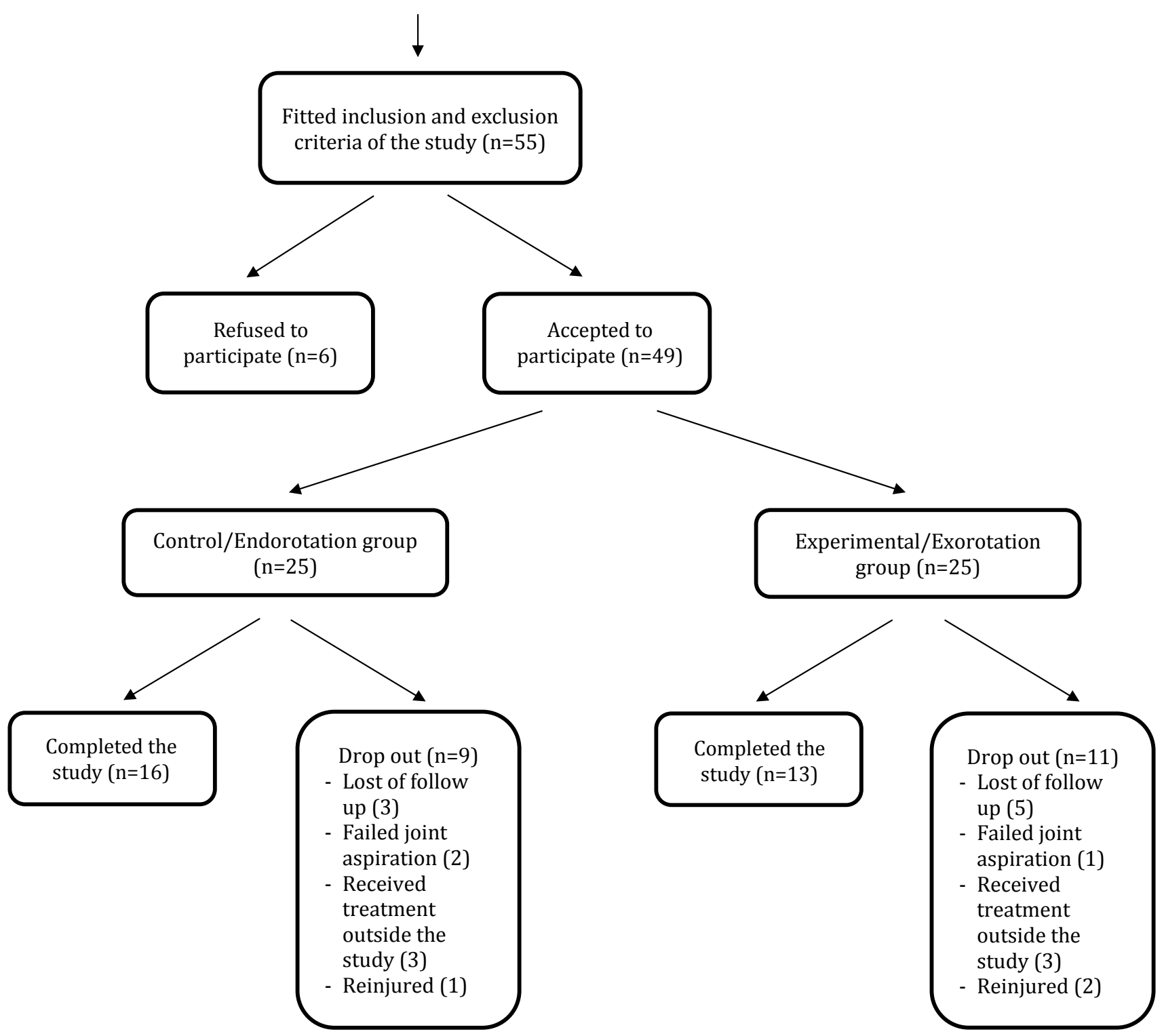

Figure 1. Schematic chart of the subjects enrolled in this study

group, tendon graft tension was changed into exorotation and fixated using BioScrew ${ }^{\circledR}$ on the medial side (Figure 2). All subjects were sent for the rehabilitation program following the standard protocol for 6 months.

\section{Tibial tubercle to trochlear groove (TTTG)}

TTTG distance is the lateral distance between the anterior tibial tubercle and trochlear groove, and it is expressed in millimeters. The TTTG distance is measured in an axial T2weighted full spin echo MRI: from the midpoint of the trochlear groove, projected into a line connecting two femoral condyles, to the most anterior point of the tibial tubercle. The TTTG distance represents tibial rotation in relation to the femur, which is altered in patients with ACL injury. The TTTG distance of more than $20 \mathrm{~mm}$ is a predictive factor for patellofemoral joint pain. ${ }^{8}$ In this study, we measured the distance and direction of TTTG. If the tibial tubercle was more medial than the trochlear groove (that is, the tibia was more endorotated to the femur), then the TTTG was negative. By contrast, if the tibia was 

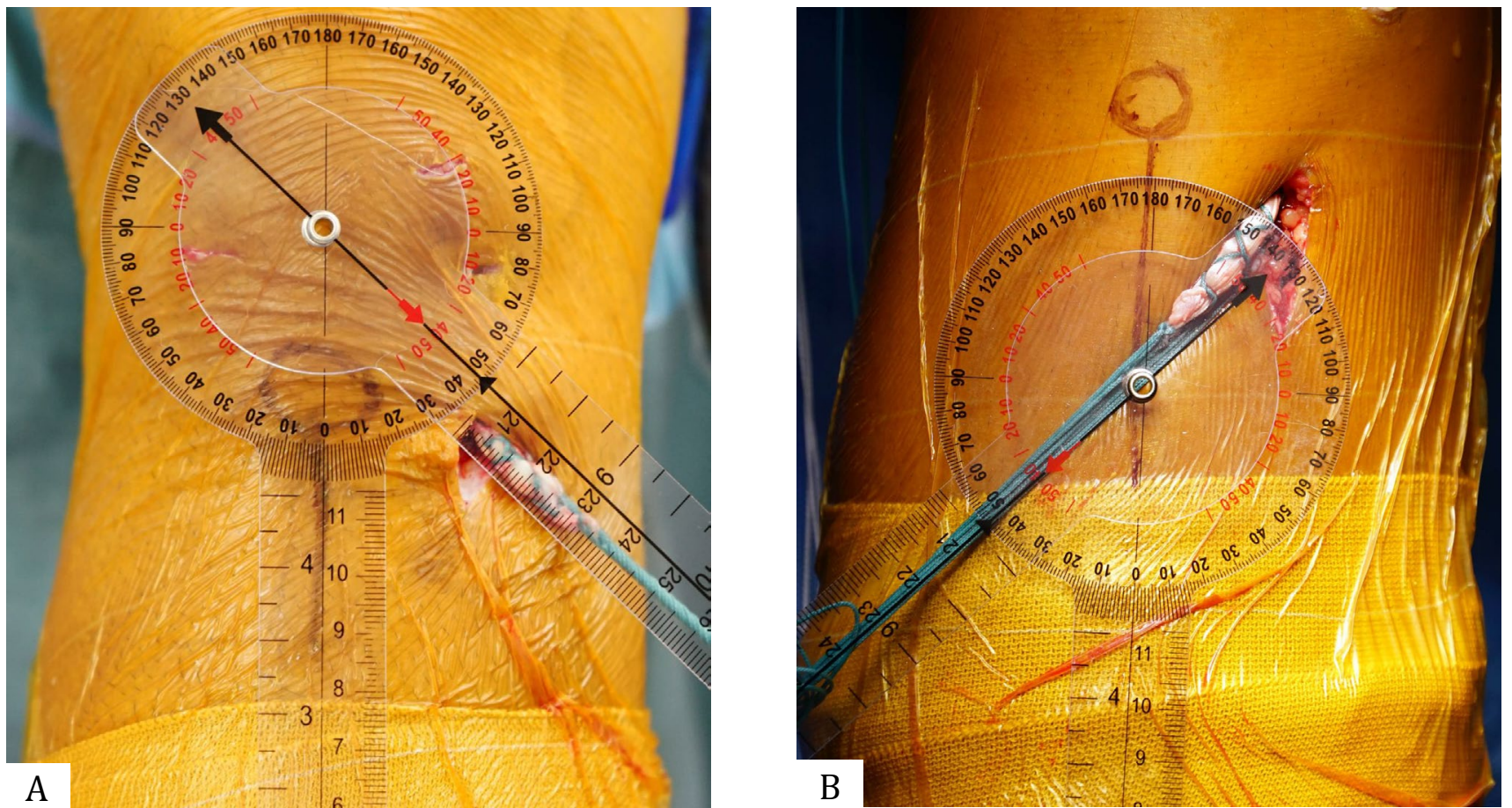

Figure 2. Difference in ACLR procedure between the study groups. This method is an intraoperative procedure of a patient with ruptured ACL of the right knee: (A) In the control group, the hamstring tendon autograft was tensioned in the direction of $45^{\circ}$ endorotation with respect to the anterior tibial crest line. (B) In the experimental group, the graft tensioning direction was modified into $45^{\circ}$ exorotation from the anterior tibial crest line
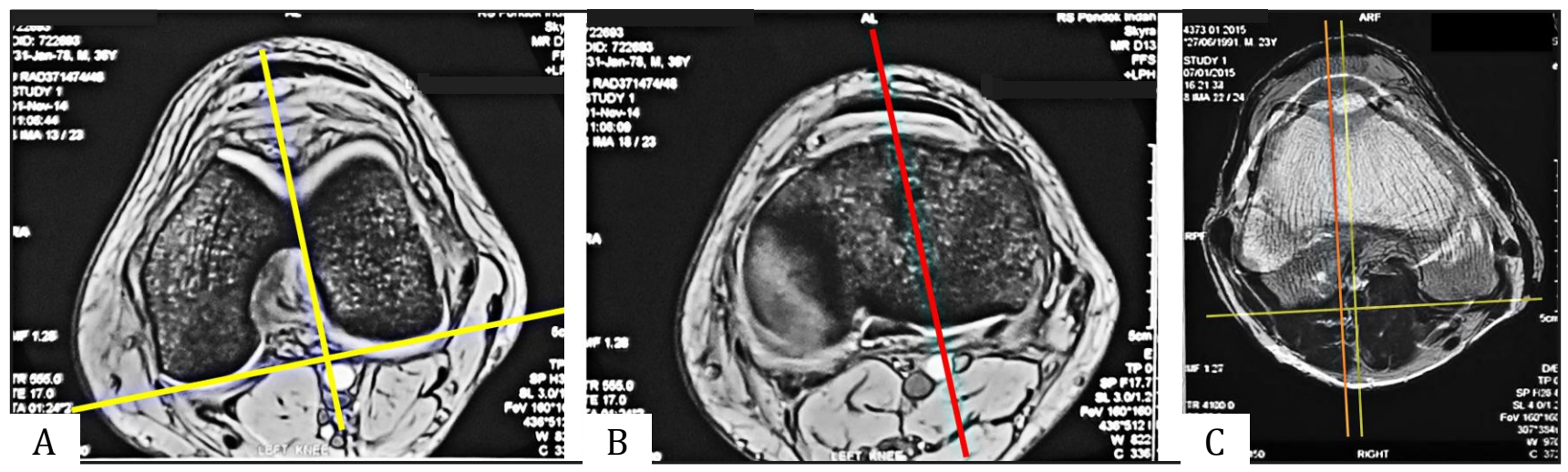

Figure 3. TTTG distance measurement. In the axial view of knee MRI, TTTG distance was measured from the midpoint of the trochlear groove, which was projected into a line connecting two femoral condyles (A), to the most anterior point of the tibial tubercle (B). The distance between two parallel lines is the TTTG distance, which represents tibial rotation in relation to the femur (C)

more exorotated in relation to the femur, then the TTTG was positive (Figure 3).

\section{Cartilage oligometric matrix protein (COMP)}

COMP is a specific non-collagen glycoprotein in the cartilage matrix. COMP in this study was obtained from synovial fluid and measured using an ELISA kit (Biovendor RD194080200). The absorbents at each well were determined using a microplate reader set at $450 \mathrm{~nm}$ and measured in nanograms per milliliter. COMP level is a non-collagen biomarker correlated for cartilage degradation ${ }^{9}$ and thereby a predictive of knee osteoarthritis.

\section{Functional outcome}

The Kujala score is a scoring system obtained from a questionnaire consisting of 13 questions documenting a patient's response to activities related to patellofemoral joint: walking, 
running, jumping, walking upstairs, squatting, and sitting with flexed knee. The maximum Kujala score is 100, and a high score indicates high patellofemoral functions.

The TTTG distance, COMP level, and Kujala score were obtained preoperatively and at 6 months postoperatively. Data were compared and analyzed using independent t-test or MannWhitney in Statistical Package for the Social Sciences (SPSS ver. 20) with a significant value of $\mathrm{p}<0.05$.

The research subjects and their families were properly informed, and written consent was obtained. Ethical clearance was obtained from the Ethical Committee of the Faculty of Medicine of Universitas Indonesia 758/UN2.F1/ETIK/2014.

\section{RESULTS}

The age range of patients was 19-35 years old $(29.40 \pm 5.39$ in the endorotation group and 25.80 \pm 5.90 in the exorotation group), and both groups consisted of mostly males. The most common cause of injury was due to futsal $(48.28 \%)$, and the duration of injury was between 6.0 and 7.5 months (Table 1 ).

\section{Effect of modified graft tension direction on TTTG}

The mean between the two groups was not significantly different before $(p=0.390)$ and after the surgery $(p=0.113)$. TTTG after surgery showed that the exorotation group had a smaller TTTG value than the endorotation group, and the difference was statistically significant $(p=0.028)$ (Table 2).

\section{Effect of modified graft tension direction on COMP}

Significant median differences were observed between the two groups before $(p=0.013)$ and after surgery $(p=0.007)$. The delta change of COMP in the exorotation group was less than that in the endorotation group $(\mathrm{p}<0.001)$ (Table 3).

\section{Effect of modification graft tension direction on the Kujala score}

Before surgery, no significant mean differences were observed between the two groups $(\mathrm{p}=0.051)$. By contrast, the results were similar after surgery $(\mathrm{p}=0.454)$. The change in the Kujala score between the two groups after surgery was statistically significant $(\mathrm{p}=0.015)$, and the exorotation group presented a higher Kujala score than the endorotation group (Table 4).

\section{Unexpected findings and adverse event}

Three patients were found to have graft rupture during follow up due to injury, which occurred between the fifth and sixth months. These three patients were excluded from this study. After 6 months, patellofemoral pain syndrome (PFPS) were found in three patients belonging to the endorotation group and one patient in the exorotation group. PFPS was identified by the presence of pain or stiffness on prolonged sitting with the knee flexed ("theater sign") and pain during stair climbing or descending. Subjects may also draw a circle with their fingers around the patella when asked to localize the pain ("circle sign"). On the basis of the TTTG values, not all patients underwent endorotation before the surgery. These three patients were then included into the exorotation group, and one of them showed PFPS after six months.

\section{DISCUSSION}

ACL rupture is most commonly caused by indirect injury that results in the twisting of the knee toward endorotation. This condition will cause an alteration in the alignment of the patellofemoral joint and often results in a patellofemoral joint dysfunction. Patellofemoral pain syndrome occurs when pain is present in the patellar region and worsens on activities that involve an increase load in patellofemoral joint such as running, jumping, climbing and descending stairs, and squatting. Crepitation is also noted during movements, and the knee presents limited range of motion. These problems will undoubtedly prevent patients with ACL injuries to return to their sporting activities.

ACLR surgery has been proven to restore anterior knee joint stability; however, problems in endorotation stability still persist. Majority of individuals who underwent ACLR are still unable to return to sports due to the inability of ACLR to significantly improve endorotation stability. Our study aimed to solve this problem by modifying 
Table 1. Subject characteristics

\begin{tabular}{|c|c|c|}
\hline & Endorotation $(n=16)$ & Exorotation $(n=13)$ \\
\hline Age (years) & $29.4 \pm 5.39$ & $25.8 \pm 5.90$ \\
\hline \multicolumn{3}{|l|}{ Gender } \\
\hline Male & 14 & 12 \\
\hline Female & 2 & 1 \\
\hline \multicolumn{3}{|l|}{ Cause of injury } \\
\hline Futsal & 9 & 5 \\
\hline Basketball & 3 & 5 \\
\hline Martial arts & 3 & 1 \\
\hline Soccer & 1 & 1 \\
\hline Kickboxing & 0 & 1 \\
\hline Onset of injury (months) & $6.9 \pm 2.28$ & $7.2 \pm 2.30$ \\
\hline
\end{tabular}

Table 2. Effect of modified graft tension direction on TTTG

\begin{tabular}{lccc}
\hline Tibial tubercle to trochlear grove (TTTG) & Endorotation $(\mathrm{n}=16)$ & Exorotation $(\mathrm{n}=13)$ & $\mathrm{p}$ \\
\hline Pre-ACLR $(\mathrm{mm})$ & $-14.8 \pm 8.94^{*}$ & $-11.8 \pm 9.57^{*}$ & 0.390 \\
Post-ACLR $(\mathrm{mm})$ & $-15.5 \pm 10.54^{*}$ & $-7.1 \pm 15.19^{*}$ & 0.113 \\
Delta change $(\delta)$ & $-2.0((-20.20)-10.80)^{\dagger}$ & $4.7 \pm 8.36$ & $\mathbf{0 . 0 2 8}$ \\
\hline
\end{tabular}

ACLR=anterior cruciate ligament reconstruction; ${ }^{*}$ negative value represents endorotation, ${ }^{\dagger}$ abnormally distributed data

Table 3. Effect of modified graft tension direction on COMP

\begin{tabular}{lccc}
\hline Cartilage oligomeric matrix protein (COMP) & Endorotation $(\mathrm{n}=16)$ & Exorotation $(\mathrm{n}=13)$ & $\mathrm{p}$ \\
\hline Pre-ACLR $(\mathrm{nm} / \mathrm{mL})$ & $17177.9(10298.7-88683.5)$ & $19318.4(11910-89385.9)$ & 0.475 \\
Post-ACLR $(\mathrm{nm} / \mathrm{mL})$ & $18453.7(8513.8-58981.4)$ & $19386(10217.8-38212.4)$ & 0.846 \\
Delta change $(\delta)$ & $1931.75(-74019-39436.6)$ & $-3224.7(-67163.2-24028.7)$ & 0.475 \\
\hline
\end{tabular}

ACLR=anterior cruciate ligament reconstruction; *negative value represents endorotation, ${ }^{\dagger}$ abnormally distributed data

Table 4. Effect of modification graft tension direction on the Kujala score

\begin{tabular}{lccc}
\hline Kujala score & Endorotation $(\mathrm{n}=16)$ & Exorotation $(\mathrm{n}=13)$ & $\mathrm{p}$ \\
\hline Pre-ACLR & $69.5(41-94)$ & $68(42-88)$ & 0.044 \\
Post-ACLR & $91(71-100)$ & $93(77-100)$ & 0.475 \\
Delta change $(\delta)$ & $17(-1-56)$ & $25(-1-43)$ & 0.476 \\
\hline
\end{tabular}

ACLR=anterior cruciate ligament reconstruction; *negative value represents endorotation, ${ }^{\dagger}$ abnormally distributed data

the graft tension direction to exorotation to reduce endorotation as compared with the standard procedure.

Patients with ACL rupture only experienced pain during the onset of the injury, but only a small number presented to the clinic due to inability to engage in high-impact activities. The problem lies in the instability of the knee joint, which is most commonly followed by movement disturbances. A small percentage of patients who seek treatment usually prefer conservative treatments, and those who undergo ACLR surgery are often unable to return to normal sporting activities. Our study showed a higher incidence of ACL rupture in males than in 
females, which might be due to the wide range of sport activities enjoyed by the male population. Males regularly engage in high-impact sports activities, such as futsal, basketball, soccer, and martial arts, whereas females tend to engage in low-impact sports. High-impact sports activities increase of ACL injury.

Futsal was the most common cause of torn ACL in this study (48.28\%). Futsal is a highly popular sports activity in Indonesia because of its low cost. A futsal field is relatively smaller than a soccer field, which translates in more opportunities for players to engage in actions that involve a great deal of knee twisting movements leading to torn ACL. Aside from futsal, basketball is the second common cause of torn ACL (27.58\%). In basketball, ACL tears are commonly caused by jumping actions, followed by landing on twisted knees. ${ }^{4}$

In this study, TTTG on MRI was used to measure the rotation of the knee. Camp et $\mathrm{al}^{10}$ and Pandit et $\mathrm{al}^{11}$ concluded that MRI is highly effective for TTTG measurements. Saper et $\mathrm{al}^{12}$ analyzed torn ACL and showed endorotation in ACL-deficient knees; Shakespeare and Fick $^{13}$ reported malalignment of the patellofemoral joint in ACL-deficient knees. The mean TTTG value before surgery in this study was 13.80 $\mathrm{mm}$. This result was similar with a previous study by Saper et $\mathrm{al}^{12}(12.70 \mathrm{~mm})$. Thus, in ACL-deficient knees, tibial endorotation occurred with respect to the femur. Postoperatively, a difference in TTTG was observed between the two study groups. The exorotation group showed significantly lower TTTG $(p<0.05)$ and more tibial exorotation than the control group. This result demonstrated that modification in the tensioning direction might restore tibiofemoral rotation in ACL-deficient knees.

COMP plays a role in the structural integrity of cartilage via its interaction with other extracellular matrix proteins such as collagens and fibronectin. COMP mediates the interaction of chondrocytes with the cartilage extracellular matrix through interactions with cell surface integrin receptors. ${ }^{14}$ It is also postulated to play a role in the pathogenesis of osteoarthritis. As a potent suppressor of apoptosis in both primary chondrocytes and transformed cells, COMP may serve as a biological marker for cartilage damage. It can be detected both in serum and synovial fluid. ${ }^{15}$
Mendias et $\mathrm{al}^{16}$ measured serum COMP levels of 18 patients with ACL injury preand postoperatively. The patients showed an increased level of serum COMP preoperatively, representing cartilage breakdown as the result of previous injury. The level of serum COMP then decreased to its almost normal level weeks after surgery. In this study, differences in COMP level changes between the two groups were noted. In the endorotation group, in which a current standard procedure of ACL reconstruction surgery was performed, the synovial COMP level increased postoperatively. By contrast, subjects in the exorotation group, who underwent modification of tensioning direction in their ACL reconstruction surgery, demonstrated a decrease in synovial COMP level postoperatively. These results represented low cartilage damage and minimal risks for associated secondary osteoarthritis in the future for the exorotation group.

At least six main causes for patellofemoral joint pain were noted: cartilage, synovial tissue, retinaculum, skin, muscle, and nerve. In cases of ACL injury, the most significant cause is patellofemoral malalignment, which can lead to imbalance of the extensor mechanism and excessive pressure on the patellofemoral joint, resulting in cartilage damage. ${ }^{17}$ ACL does not only act as anterior restrain of the knee, but it also plays a role in preventing endorotation of the knee. Several studies showed that ACLR fails to correct endorotation instability. ${ }^{18,19,20}$ Knee rotational stability in this study was measured with the TTTG distance, which was not restored by a standard technique of ACL reconstruction surgery but improved with our modification in graft tensioning direction at tibial fixation.

The Kujala score is widely used to evaluate patellofemoral joint pain..$^{21,22}$ It consists of 13 knee-specific questions, which assess patient responses to patellofemoral jointrelated disorders, including walking, running, jumping, stair climbing, squatting, swelling, and knee stiffness. Harilainen et $\mathrm{al}^{23}$ evaluated 39 patients with ACL injury at two years postoperatively and found that the Kujala score was 88 (54-100) points. Our study showed a similar result with a mean Kujala score of 89.7 points. We also measured the Kujala score before and after surgery and examined the changes 
between the two time frames. In this study, both groups showed an improvement in terms of the Kujala score. Despite improvements in the Kujala score in both groups, close inspection showed that the Kujala score in the exorotation group was significantly better than that in the endorotation group $(\mathrm{p}<0.05)$. Therefore, the modification of tension direction in ACLR surgery will result in enhanced patellofemoral joint functions after surgery.

In theory, torn ACL will result in the endorotation of the knee. ${ }^{24}$ However, in this study, a number of patients experienced exorotation after an ACL tear, which was most likely due to the position of the knee before the injury. On the basis of this finding, modification of graft tension direction to exorotation is not recommended if the knee is previously exorotated before surgery.

The present study had some limitations. The follow-up time could be prolonged up to two years to anticipate any delayed onset of anterior knee pain. With long-term follow up, the subjects may show signs and symptoms of osteoarthritis secondary to patellofemoral malalignment.

In conclusion, a new technique of modified exorotation direction of graft tension for ACL reconstructive surgery at tibial fixation showed a significant reduction in tibial internal rotation and cartilage breakdown. This technique significantly improved the functional outcome of patients with total ACL rupture.

\section{Conflicts of interest}

The authors affirm no conflict of interests in this study.

\section{REFERENCES}

1. Fu FH, van Eck CF, Tashman S, Irrgang JJ, Moreland MS. Anatomic anterior cruciate ligament reconstruction: a changing paradigm. Knee Surg Sports Traumatol Arthrosc. 2015;23(3):640-8.

2. Andersson D, Samuelsson K, Karlsson J. Treatment of anterior cruciate ligament injuries with special reference to surgical technique and rehabilitation: an assessment of randomized controlled trials. Arthroscopy. 2009;25(6):653-85.

3. Costa LA, Foni NO, Antonioli E, de Carvalho RT, Paião ID, Lenza M, et al. Analysis of 500 anterior cruciate ligament reconstructions from a private institutional register. PLoS One. 2018;13(1):e0191414.
4. Schub D, Saluan P. Anterior cruciate ligament injuries in the young athlete: evaluation and treatment. Sports Med Arthrosc Rev. 2011;19(1):34-43.

5. Cheatham SA, Johnson DL. Anatomic revision ACL reconstruction. Sports Med Arthrosc Rev. 2010;18(1):33-9.

6. Lyman S, Koulouvaris P, Sherman S, Do H, Mandl LA, Marx RG. Epidemiology of anterior cruciate ligament reconstruction: trends, readmissions, and subsequent knee surgery. J Bone Joint Surg Am. 2009;91(10):2321-8.

7. Van de Velde SK, Gill TJ, DeFrate LE, Papannagari R, Li G. The effect of anterior cruciate ligament deficiency and reconstruction on the patellofemoral joint. Am J Sport Med. 2008;36(6):1150-9.

8. Osman NM, Ebrahim SM. Patellofemoral instability: quantitative evaluation of predisposing factors by MRI. Egypt J Radiol Nucl Med. 2016;47(4):1529-38.

9. Das Gupta E, Ng WR, Wong SF, Bhurhanudeen AK, Yeap SS. Correlation of serum cartilage oligometric matrix protein (COMP) and interleukin-16 (IL-16) levels with disease severity in primary knee osteoarthritis: a pilot study in a Malaysian population. PLoS One. 2017;12(9):e0184802.

10. Camp CL, Stuart MJ, Krych AJ, Levy BA, Bond JR, Collins MS, et al. CT and MRI measurements of tibial tubercle-trochlear groove distances are not equivalent in patients with patellar instability. Am J Sports Med. 2013;41(8):1835-40.

11. Pandit S, Frampton C, Stoddart J, Lynskey T. Magnetic resonance imaging assessment of tibial tuberositytrochlear groove distance: normal values for males and females. Int Orthop. 2011;35(12):1799-803.

12. Saper MG, Popovich JM, Fajardo R, Hess S, Pascotto JL, Shingles $M$. The relationship between tibial tubercletrochlear groove distance and noncontact anterior cruciate ligament injuries in adolescents and young adults. Arthroscopy. 2016;32(1):63-8.

13. Shakespeare D, Fick D. Patellar instability-can the TT-TG distance be measured clinically? Knee. 2005;12(3):201-4.

14. Neidhart M, Hauser N, Paulsson M, DiCesare PE, Michel BA, Häuselmann HJ. Small fragments of cartilage oligomeric matrix protein in synovial fluid and serum as markers for cartilage degradation. Br J Rheumatol. 1997;36(11):1151-60.

15. Streich NA, Zimmermann D, Schmitt H, Bode G. Biochemical markers in the diagnosis of chondral defects following anterior cruciate ligament insufficiency. Int Orthop. 2011;35(11):1633-7.

16. Mendias CL, Lynch EB, Davis ME, Enselman ER, Harning JA, DeWolf PD, et al. Changes in circulating biomarkers of muscle atrophy, inflammation, and cartilage turnover in patients undergoing anterior cruciate ligament reconstruction and rehabilitation. Am J Sports Med. 2013;41(8):1819-26.

17. Smith TO, Davies L, O'Driscoll ML, Donell ST. An evaluation of the clinical tests and outcome measures used to assess patellar instability. Knee. 2008;15(4):255-62.

18. Yagi M, Kuroda R, Nagamune K, Yoshiya S, Kurosaka M. Double-bundle ACL reconstruction can improve rotational stability. Clin Orthop Relat Res. 2007;454:100-7.

19. Mihelic R, Jurdana H, Jotanovic Z, Madjarevic T, Tudor 
A. Long-term results of anterior cruciate ligament reconstruction: a comparison with non-operative treatment with a follow-up of 17-20 years. Int Orthop. 2011;35(7):1093-7.

20. Leys T, Salmon L, Waller A, Linklater J, Pinczewski L. Clinical results and risk factors for reinjury 15 years after anterior cruciate ligament reconstruction: a prospective study of hamstring and patellar tendon grafts. Am J Sports Med. 2012;40(3):595-605.

21. Kuru T, Dereli EE, Yaliman A. Validity of the Turkish version of the Kujala patellofemoral score in patellofemoral pain syndrome. Acta Orthop Traumatol Turc. 2010;44(2):152-6.

22. Chiu JK, Wong YM, Yung PS, Ng GY. The effects of quadriceps strengthening on pain, function, and patellofemoral joint contact area in persons with patellofemoral pain. Am J Phys Med Rehabil. 2012;91(2):98-106.

23. Harilainen A, Linko E, Sandelin J. Randomized prospective study of ACL reconstruction with interference screw fixation in patellar tendon autografts versus femoral metal plate suspension and tibial post fixation in hamstring tendon autografts: 5-year clinical and radiological follow-up results. Knee Surg Sports Traumatol Arthrosc. 2006;14(6):517-28.

24. Oliveira JP, Fonseca F, Noronha JC. Anterior knee pain and sensitivity after anterior cruciate ligament: its impact on sports. OA Orthopaedics. 2013;1(2):13. 